\title{
Hypotension and hypocapnia during general anesthesia in piglets: study of S100b as an acute biomarker for cerebral tissue injury
}

Clausen, Nicola G ; Antonsen, Steen ; Spielmann, Nelly ; Hansen, Tom G ; Weiss, Markus ; Ringer, Simone K

\begin{abstract}
BACKGROUND: Hypotension and/or hypocapnia might increase general anesthesia (GA)-related neuromorbidity in infants, but safe levels of perioperative blood pressure are poorly defined. Serum protein S100b has been used as screening, monitoring, and prediction tool in the management of patients with traumatic brain injury. Using an animal model, we investigated serum S100b as an acute biomarker of cerebral hypoperfusion and cerebral cell dysfunction during hypotension, hypocapnia, or combined hypotension/hypocapnia during GA. METHODS: Fifty-seven sevoflurane-midazolam anesthetized piglets aged 4 to 6 weeks were randomly allocated to control $(n=9)$, hypotension $(n=18)$, hypocapnia $(n=20)$, or combined hypotension and hypocapnia $(n=10)$. Hypotension (target mean arterial blood pressure: 35 to 38 or 27 to $30 \mathrm{~mm} \mathrm{Hg}$ ) was induced by blood withdrawal and nitroprusside infusion, and hypocapnia by hyperventilation (target PaCO2: 28 to 30 and 23 to $25 \mathrm{~mm} \mathrm{Hg}$ ). Serum S100b and albumin were measured at baseline, before and 60 minutes after the interventions, and following 60-minute recovery. RESULTS: Serum S100b concentrations decreased over time $(\mathrm{P}=0.001)$, but there was no difference in S100b between control piglets and those exposed to hypotension, hypocapnea, or a combination of the both $(\mathrm{P}=0.105)$. Albumin decreased in all 4 groups $(\mathrm{P}=0.001)$. CONCLUSION: S100b did not increase following 60 minutes of systemic hypotension and/or hypocapnia during GA in piglets. In this setting, the use of S100b as a biomarker of cerebral cell tissue dysfunction cannot be supported.
\end{abstract}

DOI: https://doi.org/10.1097/ANA.0000000000000601

Posted at the Zurich Open Repository and Archive, University of Zurich

ZORA URL: https://doi.org/10.5167/uzh-175922

Journal Article

Published Version

Originally published at:

Clausen, Nicola G; Antonsen, Steen; Spielmann, Nelly; Hansen, Tom G; Weiss, Markus; Ringer, Simone K (2020). Hypotension and hypocapnia during general anesthesia in piglets: study of S100b as an acute biomarker for cerebral tissue injury. Journal of Neurosurgical Anesthesiology, 32(3):273-278.

DOI: https://doi.org/10.1097/ANA.0000000000000601 


\title{
Hypotension and Hypocapnia During General Anesthesia in Piglets: Study of S100b as an Acute Biomarker for Cerebral Tissue Injury
}

\author{
Nicola G. Clausen, MD, PhD, *† Steen Antonsen, MD, PhD, $\S$ Nelly Spielmann, MSc, $\neq$ \\ Tom G. Hansen, MD, PhD, * Markus Weiss, MD, DMSc, † and Simone K. Ringer, DVM, PhD\|
}

\begin{abstract}
Background: Hypotension and/or hypocapnia might increase general anesthesia (GA)-related neuromorbidity in infants, but safe levels of perioperative blood pressure are poorly defined. Serum protein S100b has been used as screening, monitoring, and prediction tool in the management of patients with traumatic brain injury. Using an animal model, we investigated serum S100b as an acute biomarker of cerebral hypoperfusion and cerebral cell dysfunction during hypotension, hypocapnia, or combined hypotension/hypocapnia during GA.
\end{abstract}

Methods: Fifty-seven sevoflurane-midazolam anesthetized piglets aged 4 to 6 weeks were randomly allocated to control $(n=9)$, hypotension $(n=18)$, hypocapnia $(n=20)$, or combined hypotension and hypocapnia $(\mathrm{n}=10)$. Hypotension (target mean arterial blood pressure: 35 to 38 or 27 to $30 \mathrm{~mm} \mathrm{Hg}$ ) was induced by blood withdrawal and nitroprusside infusion, and hypocapnia by hyperventilation (target $\mathrm{PaCO}_{2}$ : 28 to 30 and 23 to $25 \mathrm{~mm} \mathrm{Hg}$ ). Serum S100b and albumin were measured at baseline, before and 60 minutes after the interventions, and following 60-minute recovery.

Results: Serum S100b concentrations decreased over time $(P=0.001)$, but there was no difference in $\mathrm{S} 100 \mathrm{~b}$ between control piglets and those exposed to hypotension, hypocapnea, or a combination of the both $(P=0.105)$. Albumin decreased in all 4 groups $(P=0.001)$.

Conclusion: S100b did not increase following 60 minutes of systemic hypotension and/or hypocapnia during GA in piglets. In this setting, the use of S100b as a biomarker of cerebral cell tissue dysfunction cannot be supported.

Received for publication September 9, 2018; accepted March 14, 2019. From the Departments of *Anesthesiology and Intensive Care, Odense University Hospital, Odense; §Clinical Biochemistry, Odense University Hospital, Svendborg, Denmark; †Children's Research Center; †Department of Anesthesiology, University Children's Hospital; and \|Section Anesthesiology, Vetsuisse Faculty, University of Zurich, Zurich, Switzerland.

The study is part of Mrs. Clausen's PhD project, which was awarded the ESA MAQUET GRANT at the annual meeting of the European Society of Anesthesia 2015. Mrs Clausen's project is further supported by funds provided by the Department of Anesthesiology and Intensive Care Medicine, University Hospital Odense.

The authors have no conflicts of interest to disclose.

Address correspondence to: Nicola G. Clausen, MD, PhD, E-mail: nicola@nicola.dk.

Supplemental Digital Content is available for this article. Direct URL citations appear in the printed text and are provided in the HTML and PDF versions of this article on the journal's website, www.jnsa.com.

Copyright (C) 2019 Wolters Kluwer Health, Inc. All rights reserved.

DOI: 10.1097/ANA.0000000000000601
Key Words: anesthesia, general, cerebral circulation, vascular hypotension, domestic pig, biologic marker, S100 calcium binding protein beta subunit

(J Neurosurg Anesthesiol 2019;00:000-000)

$T$ he risks associated with general anesthesia (GA) in children are increasingly acknowledged. Recently, the APRICOT study reported a $5.2 \%$ incidence of critical adverse events in pediatric anesthesia practice in Europe. ${ }^{1}$ Fifty-five percent of the cardiovascular adverse events reported in this study were arterial hypotension. Perianesthetic arterial hypotension has previously been found to be associated with decreased regional cerebral oxygen saturation measured by near-infrared spectroscopy. ${ }^{2}$ In a preclinical model, our group recently showed that cerebral tissue partial pressure of oxygen decreased significantly when blood pressure was lowered to 27 to 30 or 35 to $38 \mathrm{~mm} \mathrm{Hg}{ }^{3}$ In a clinical pediatric setting, however, there are currently no reliable means to estimate neuronal injury during GA. Glial protein S100b is clinically used as a biomarker for characterizing mild traumatic brain injury ${ }^{4}$ and has been associated with perioperative neuromorbidity in neonates undergoing cardiac surgery. ${ }^{5}$

We hypothesized that systemic hypotension and hypocapnia in piglets, either alone or in combination, would reduce cerebral perfusion and may cause neuronal cell damage which might be reflected in an increase in serum levels of S100b. The aim of this study was to assess any association between cerebral hypoperfusion during GA and serum levels of S100b. In a pediatric piglet model, we compared serum levels of S100b and albumin (to estimate hemodilution) during stable GA and GA with temporary hypotension, hypocapnia or combined hypotension, and hypocapnia.

\section{METHODS}

The study was approved by the local Ethics Committee for Animal Experiments (License number ZH175/16). We chose 4- to 6-week-old piglets to test our hypothesis because, at that age, the pig brain is similar to infants in morphology and development. ${ }^{6}$

Fifty-seven, 4- to 6-week-old piglets (breed: pure largewhite, Swiss landrace or duroc, or crossings of the 3) were included in the study. The piglets were housed in their home 
facility until transportation in boxes padded with straw and side openings for ventilation. The piglets were randomly allocated to 1 of 4 groups: control $(C)(n=9)$, moderate or severe hypotension $(\mathrm{HT})(\mathrm{n}=18)$, moderate or severe hypocapnia (HC) $(n=20)$, or moderate hypotension and hypocapnia combined (HTC) $(n=10)$. All animals were anesthetized according to an identical protocol (see below). A supplement outlining the study compliance with the Animal Research: Reporting in Vivo Experiments guidelines is provided in the supplementary material (Supplemental Digital Content 1, http://links.lww.com/JNA/A116). ${ }^{7}$

\section{Anesthesia and Instrumentation}

This study was part of our aforementioned study, and the experimental protocol is described in detail elsewhere. ${ }^{3}$ In brief, after mask induction of anesthesia with sevoflurane in oxygen, the animals were continuously monitored with pulse oximetry, expired gases, including end-tidal carbon dioxide $\left(\mathrm{EtCO}_{2}\right)$, spirometry, electrocardiogram, rectal temperature, and invasive blood pressure by a 22-G catheter (BD Insyte-A, VD Vialon Material; Becton Dickinson, Allschwil, BL, Switzerland) inserted into the femoral artery. Two intravenous (IV) catheters were placed in ear veins. Without administration of a muscle relaxant, the animals were orotracheally intubated with a cuffed tube size 4.0 (Microcuff; Kimberly-Clark $\mathrm{GmbH}$, Niederbipp, Switzerland) while placed in the sternal position. The animals were mechanically ventilated (Avance CS2; Anandic Medical Systems AG, Feuerthalen, Switzerland) throughout the experiment. Anesthesia was maintained with sevoflurane in oxygen/air mixture and a continuous infusion of midazolam $(1 \mathrm{mg} / \mathrm{kg} / \mathrm{h})$. After administration of an IV bolus of fentanyl $(10 \mu \mathrm{g} / \mathrm{kg})$ and local infiltration with lidocaine $0.1 \mathrm{~mL}$ and bupivacaine $0.1 \mathrm{~mL}$, the piglets were instrumented with frontal craniotomy bolts to allow monitoring of cerebral tissue partial pressure of oxygen and cerebral blood flow. A single operator (N.G.C.) performed these procedures. Data from these intracranial monitoring devices were included in our previously published study ${ }^{3}$ and are not relevant to the current study.

An experienced veterinarian anesthetist (S.K.R.) continuously assessed the animals. Normoxia, normocapnia, normoglycemia, and normothermia were maintained and the piglets were hydrated with an IV infusion of Ringer's acetate with glucose $1 \%(5 \mathrm{~mL} / \mathrm{kg} / \mathrm{h})$. Unintended arterial hypotension (mean arterial blood pressure [MAP] $<50 \mathrm{~mm} \mathrm{Hg}$ ) was treated with a norepinephrine infusion starting at $0.03 \mu \mathrm{g} / \mathrm{kg} / \mathrm{min}$ and titrated according to effect.

\section{Study Protocol}

Two hours after instrumentation, stable physiological parameters were confirmed and the experimental protocol was initiated. In the HT group, hypotension was induced by withdrawal of arterial blood $(10 \mathrm{~mL} / \mathrm{kg}$ body weight over 5 $\mathrm{min}$ ) and nitroprusside infusion (starting dose $6 \mu \mathrm{g} / \mathrm{kg} / \mathrm{min}$, titrated to effect). In animals randomized to the HT group, norepinephrine infusion was discontinued if it had previously been started to maintain MAP. Target MAP values in the HT group were 35 to $38 \mathrm{~mm} \mathrm{Hg}$ for moderate hypotension and 27 to $30 \mathrm{~mm} \mathrm{Hg}$ for severe hypotension. Once the target MAP had been reached, blood pressure and anesthesia were maintained stable for 60 minutes. Subsequently, normotension was restored by the discontinuation of nitroprusside infusion and restoration of the volume of blood withdrawn with balanced hydroxyethyl starch 130/0.4. In the HC group, hypocapnia was induced by increasing the ventilation rate and tidal volume. Target $\mathrm{PaCO}_{2}$ values were 28 to 30 and 23 to $25 \mathrm{~mm} \mathrm{Hg}$ for moderate and severe hypocapnia, respectively. In piglets allocated to the HTC group, moderate hypotension and hypocapnia were induced simultaneously using the protocols described previously. Each intervention was followed by a 60-minute recovery period with stable vital parameters within normal ranges. Anesthesia in control animals was kept stable for identical timeframes. After final blood sampling, including arterial blood gases, the piglets were euthanized with T61 euthanasia solution.

\section{Blood Sampling and Analyses}

Venous and arterial blood samples were collected for measurement of S100b and albumin (to estimate hemodilution) at the following timepoints: after induction of anesthesia and instrumentation (baseline [B]), at the start [Tr0], and end of the treatment intervention [Tr60] (i.e., HT, HC, or HTC), and after 60 minutes of recovery with normal vital signs [post-Tr60] (Fig. 1). The samples were left to clot for 60 minutes, centrifuged at $1400 \mathrm{~g}$ for 7 minutes, frozen and subsequently stored at $-80^{\circ} \mathrm{C}$ until analysis. On the day of analysis, the samples were thawed, centrifuged, and the tests performed on the supernatants. All 4 samples from the same animal were analyzed on the same day, and venous samples were preferred. If $\geq 1$ venous sample was missing, then arterial samples were used for all measurement points in that animal.

$\mathrm{S} 100 \mathrm{~b}$ and albumin were measured on the same blood sample. S100b was measured by chemiluminescence immunoassay (Cobas e602; Roche Diagnostics, Roche a/s, Hvidovre-Copenhagen, Denmark). Albumin was analyzed by colorimetry using bromocresolgreen (Cobas c702; Roche Diagnostics). The intra-assay and interassay coefficients of variability of samples from the piglets were $2.1 \%$ and $1.4 \%$, respectively, for $\mathrm{S} 100 \mathrm{~b}$ and $3.9 \%$ and $1.3 \%$, respectively, for albumin.

\section{Statistical Analysis}

Data were analyzed at baseline (B), at the start of treatment $(\operatorname{Tr} 0)$, after 60 minutes of treatment $(\operatorname{Tr} 60)$, and after 60 minutes of recovery (post-Tr60) using IBM SPSS Statistics Version 22 (IBM Corp) and GraphPad Prism 5 for Mac OS X software. Kolmogorov-Smirnov and Saphiro-Wilk were used to test for normality distribution. Repeated measures mixed ANOVA followed by Sidak and Bonferroni post hoc tests were used to investigate changes over time and between groups. All data are presented as mean \pm SD. A $P$-value $<0.05$ was considered statistically significant.

\section{RESULTS}

All 57 piglets completed the study without any adverse events, and the intended treatment interventions were delivered in all animals. Demographics are presented in Table 1. S100b and albumin at the various study 


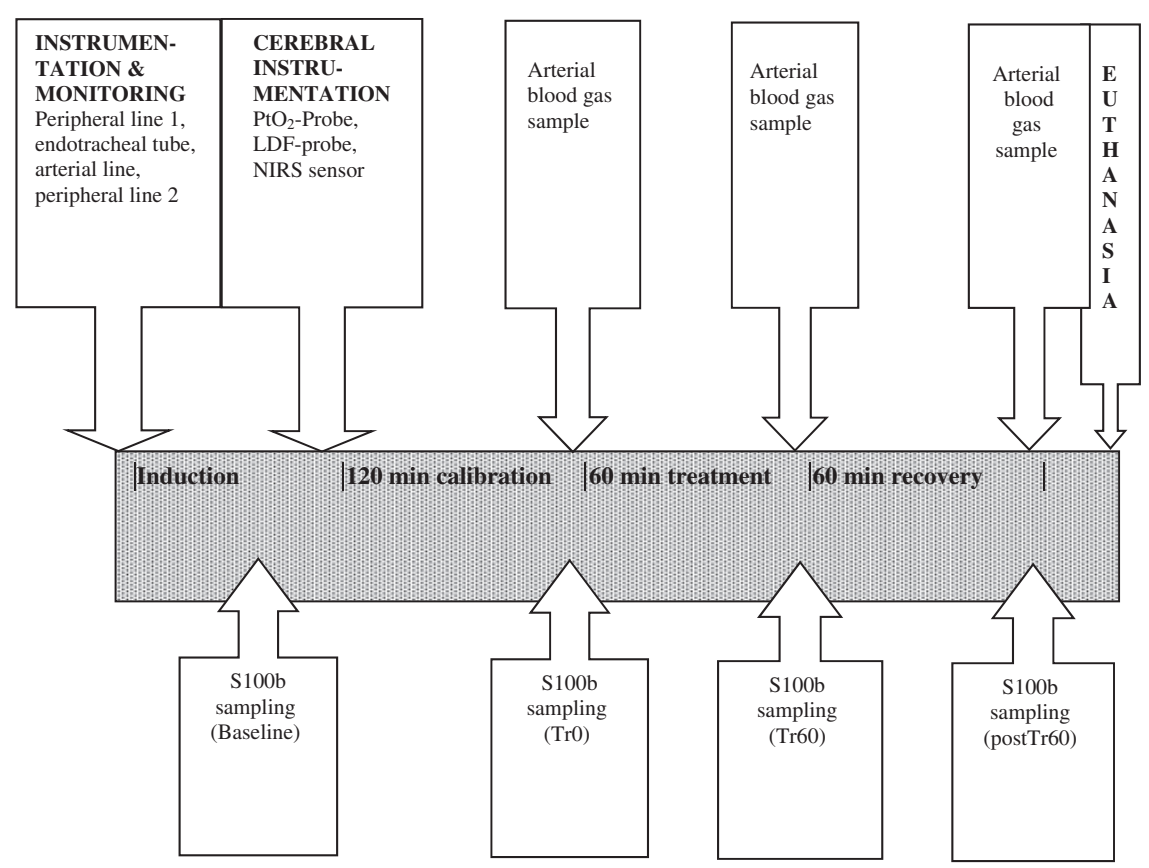

FIGURE 1. Schematic of the experimental outline and timepoints for S100b/albumin and arterial blood gas sampling. Baseline was defined as the timepoint after induction and completed monitoring but before cerebral instrumentation with a tissue partial oxygen pressure and Doppler flow probe.

timepoints (B, Tr0, $\operatorname{Tr} 60$, and post-Tr60) are shown in Figure 2, and individual serum levels of S100b and albumin (for control and treatment groups) in supplementary material (Supplementary Figs. 2 and 3, Supplemental Digital Content 2, http://links.lww.com/JNA/A117 and Supplemental Digital Content 3, http://links.lww.com/JNA/A118).

In all 4 groups, $\mathrm{S} 100 \mathrm{~b}$ concentrations decreased over time $(P=0.001)$ independent of interventions $(P=0.105)$. Similar changes were seen when arterial samples were excluded from the analysis (data not shown). The S100b pattern differed substantially in piglet number 13 (group HT): S100b at baseline was $1.11 \mu \mathrm{g} / \mathrm{L}$, peaked at $\operatorname{Tr} 0(3.99 \mu \mathrm{g} / \mathrm{L})$, and then decreased. Tr60 and post-Tr60 values remained comparatively high (3.26 and $2.93 \mu \mathrm{g} / \mathrm{L}$, respectively). To avoid inclusion of results based on erroneous measurements, the samples of piglet 13 were retested and the results remained unchanged. As piglet 13 and its anesthetic course did not differ notably from the other animals, these data were included in the statistical analysis.

S100b was primarily analyzed in venous blood samples. However, in 5 piglets, venous blood could not be withdrawn and arterial blood samples were used for analysis. To check for differences in S100b levels according to blood specimen type, we measured S100b in both arterial and venous blood samples in 3 random piglets. The mean difference was $14 \%$ with arterial S100b lower than venous. Therefore, inferential statistics were repeated excluding data from the 5 piglets in whom venous blood could not be withdrawn $(\mathrm{n}=1$ group $\mathrm{C}, \mathrm{n}=1$ group $\mathrm{HC}$, and $n=3$ group HT). Overall, the results were unchanged.

TABLE 1. Demographics and Study Characteristics

\begin{tabular}{lccccc}
\hline & Overall & Controls & Hypotension & Hypocapnia & Hypocapnia+Hypotension \\
\hline No. piglets & 57 & 9 & 18 & 20 & 10 \\
Mean age \pm SD (d) & $33.5 \pm 5.9$ & $30.8 \pm 6.7$ & $34.9 \pm 5.7$ & $33.3 \pm 5.4$ & $34.1 \pm 5.5$ \\
Mean weight \pm SD (kg) & $6.6 \pm 0.7$ & $6.6 \pm 0.8$ & $6.5 \pm 0.7$ & $6.4 \pm 0.7$ & $6.9 \pm 0.7$ \\
Norepinephrine infusion at baseline (\%) & 50.9 & 77.8 & 55.6 & 45.0 & 30.0 \\
Norepinephrine infusion after treatment (\%) & 66.7 & 77.8 & 66.7 & 70.0 & 50.0 \\
Anesthesia time (mean \pm SD) (min)* & $220.5 \pm 35.7$ & $211.9 \pm 37.8$ & $236.1 \pm 44.3$ & $210.9 \pm 26.0$ & $219.6 \pm 20.2$ \\
Anesthesia time total (mean \pm SD) (min) $\dagger$ & $343.7 \pm 37.9$ & $338.8 \pm 33.6$ & $360.9 \pm 51.1$ & $333.9 \pm 24.6$ & $337.0 \pm 22.0$
\end{tabular}

Columns: study characteristics for total study population and per study group.

Rows: number of piglets; piglets mean age \pm SD; piglets mean weight \pm SD; proportion of piglets (in percent) requiring infusion of norepinephrine to maintain mean arterial pressure of $\geq 50 \mathrm{~mm} \mathrm{Hg}$ at timepoints baseline and $\operatorname{Tr} 60$; mean duration of anesthesia in minutes from time of induction to start of treatment $(\operatorname{Tr} 0) \pm \mathrm{SD}$ (variations because of different length of time used for instrumentation and adjustment to target mean arterial pressure according to the allocated treatment group); mean duration of anesthesia in minutes from time of induction to end of recovery (post-Tr60).

*Induction of anesthesia to start of treatment $(\operatorname{Tr} 0)$

$\dagger$ Induction of anesthesia to end of recovery (post-Tr60).

Copyright (C) 2019 Wolters Kluwer Health, Inc. Unauthorized reproduction of this article is prohibited. This paper can be cited using the date of access and the unique DOI number which can be found in the footnotes. 

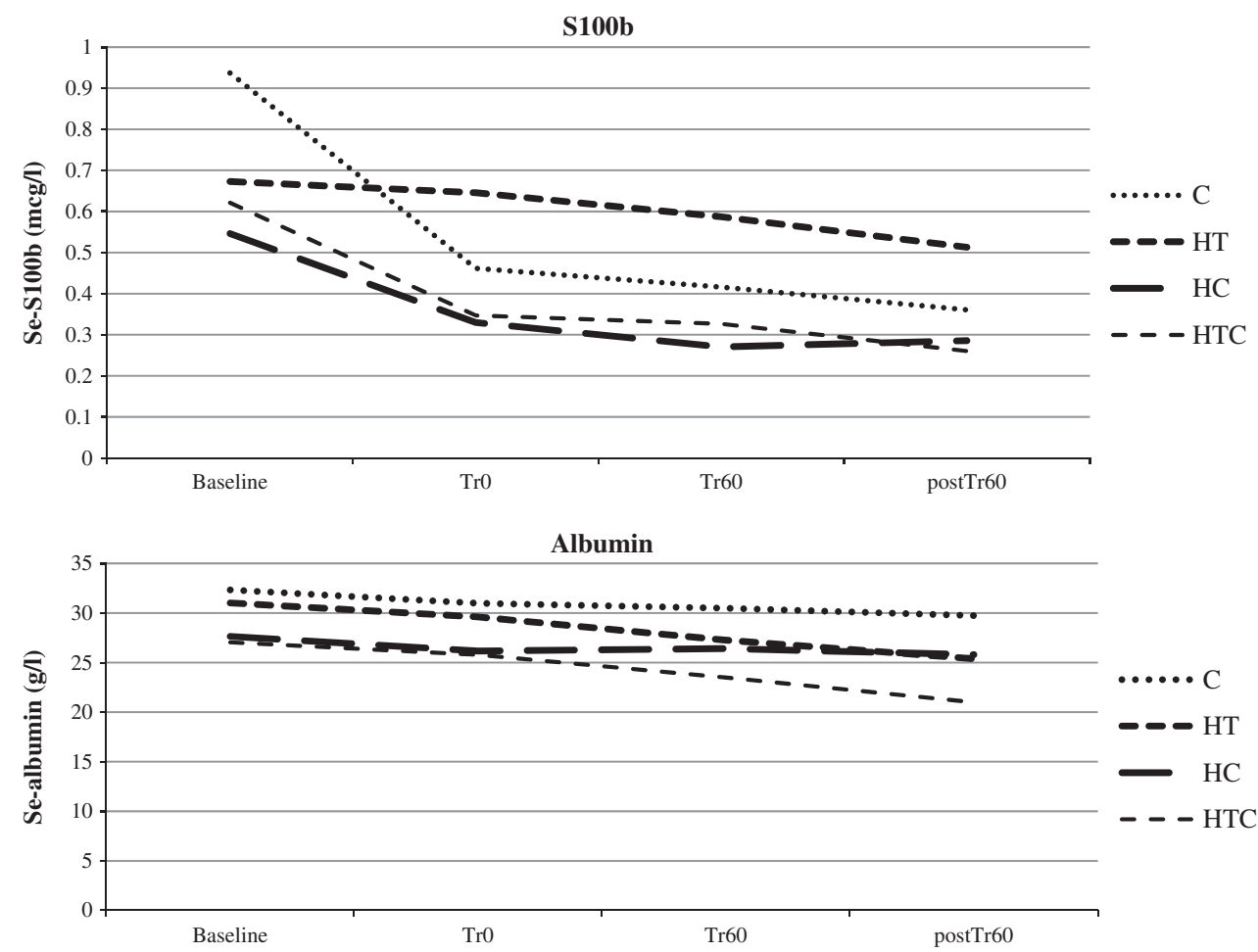

FIGURE 2. Repeated measures of S100b and albumin at timepoints baseline (B), treatment start (Tr0), treatment end (Tr60), and end of recovery (post-Tr60). Serum levels shown for controls and treatment groups. S100b is presented as $\mu \mathrm{g} / \mathrm{L}$ and albumin as $\mathrm{g} / \mathrm{L}$. $\mathrm{C}$ indicates control; HC, hypocapnia; HT, hypotension; HTC, hypotension/hypocapnia groups.

Only at timepoint post-Tr60, did S100b differ significantly between the combined HTC group and piglets treated with HC alone $\left(P_{\text {including_art }}=0.038, P_{\text {excluding_art }}=0.65\right)$. The actual S100b differences, however, were minor (mean \pm $\mathrm{SD}, 0.327 \pm 0.091$ in group HTC and $0.416 \pm 0.128$ in group HC), and unlikely to be of clinical relevance.

Albumin decreased from baseline to post-Tr60 independent of intervention $(P=0.001)$. However, some statistical differences were detected between treatment groups: at timepoints $\operatorname{Tr} 60$ and post-Tr60, albumin was lower in the group HTC compared with controls $(P=0.001)$. Further, albumin in the group HTC differed significantly from that in the group $\mathrm{HC}$ at the end of recovery $(P=0.038)$. However, after exclusion of arterial samples, this difference was no longer significant $(P=0.65)$.

\section{DISCUSSION}

In piglets, neither GA with sevoflurane and midazolam nor hypotension and/or hypocapnia triggered an increase in serum S100b. From induction of anesthesia onwards, S100b decreased in all piglets irrespective of treatment, including in the control animals. Adjusting S100b results for dilution by comparison with relative changes in albumin did not change this pattern (data not shown).

Ringer et $\mathrm{al}^{8}$ exposed piglets to hypotension or hypocapnia during sevoflurane anesthesia and demonstrated signs of neuronal dysfunction and early ischemia on magnetic resonance imaging. As an extension of this model, we investigated the association between periodic hypotension and brain tissue oxygen partial pressure, regional cerebral oxygen saturation, or cerebral blood flow in a previous study. ${ }^{3}$ Although no changes in brain tissue oxygen partial pressure were detected over time in the control group, a significant decrease in partial pressure was observed immediately after the induction of hypotension. In addition, base excess was reduced (mean $\pm \mathrm{SD}$ at baseline, $4.8 \pm 1.85$ vs. $2.0 \pm 2.65 \mathrm{mmol} / \mathrm{L}$ at $\operatorname{Tr} 60$ ) and serum lactate increased (mean $\pm \mathrm{SD}, 1.5 \pm 0.42$ vs. $2.4 \pm 0.9 \mathrm{mmol} /$ L) suggesting severe systemic hypoperfusion.

In an adult pig model, linear changes between $\mathrm{EtCO}_{2}$ and brain tissue oxygen partial pressure were shown within the range of 20 to $60 \mathrm{~mm} \mathrm{Hg}$. ${ }^{9}$ Similarly, brain tissue oxygen partial pressure was reduced by $17 \%$ and $57 \%$, respectively, following moderate $\left(\mathrm{EtCO}_{2} 30 \mathrm{~mm} \mathrm{Hg} / 4.0 \mathrm{kPa}\right)$ and severe $\left(\mathrm{EtCO}_{2} 20 \mathrm{~mm} \mathrm{Hg} / 2.7 \mathrm{kPa}\right)$ hyperventilation. ${ }^{10}$ These results indicate that perianesthetic hypotension and/ or hypocapnia reduce cerebral oxygenation.

In the normal developing brain, and following acute glial activation in response to injury, extracellular S100b has beneficial trophic activities and acts as a neuronal survival protein. ${ }^{1}$ In contrast, S100b at high concentrations is known to be directly neurotoxic and associated with poor prognosis of chronic neuroinflammatory diseases. ${ }^{12}$ A decrease in S100b as detected in our experiment might be explained by dysfunction of a glial activation cycle. ${ }^{12}$

Besides reflecting injury to neuroglia, $\mathrm{S100b}$ has been suggested as a marker of blood-brain barrier (BBB)

\section{4 | www.jnsa.com}

Copyright $(2019$ Wolters Kluwer Health, Inc. All rights reserved.

Copyright (C) 2019 Wolters Kluwer Health, Inc. Unauthorized reproduction of this article is prohibited. This paper can be cited using the date of access and the unique DOI number which can be found in the footnotes. 
integrity. ${ }^{13}$ S100b is hydrophilic and does not easily penetrate the tight junctions of the BBB. Using a model identical to ours, Ringer et $\mathrm{al}^{8}$ were able to demonstrate signs of neuronal dysfunction and ischemia. Thus, we assume that the injury they identified would be similar to that in the current study. However, the impact of the interventions in our study might have been too small to allow for any systemic leakage of intracerebral S100b through the BBB. This somewhat contradicts the fact that $\mathrm{S} 100 \mathrm{~b}$ can be elevated even in mild head trauma with discrete clinical symptoms (Glasgow Coma Score 14 or 15). ${ }^{14}$

Recent research suggests that S100b does not leak from cerebrospinal fluid to serum through breaches in the BBB. Rather, it is released from astrocytes through aquaporin-4 receptors into the Virchow-Robin space, a pia-lined interstitial fluid-filled space in the brain that surrounds vessels perforating deep into the brain white matter. Metabolites from neurons and glia cells are cleared from this space by "convective glymphatic flow" and drained to the venous circulation through the cervical lymph nodes. ${ }^{15,16}$ The flow through the glymphatic system is driven by arterial pulsation. The fluid volume in the cerebral perivascular space increases during sleep and GA but decreases in the awake state. This could explain why S100b levels in serum decreased at all timepoints independent of treatment group in our study - even in the control group, where cerebral perfusion would not be expected to be compromised. Several studies have reported neuroprotective effects of inhalational anesthetics in both in vitro and preclinical models. ${ }^{17,18}$ It could be speculated that this compensation is because of increased glymphatic flow rather than an effect of the anesthetic per se. Either way, sevoflurane may compensate for a damaging effect caused by hypotension and/or hypocapnia.

$\mathrm{S} 100 \mathrm{~b}$ is clinically used as a biomarker to categorize the severity of adult traumatic brain injury. ${ }^{4}$ In a recent update of the Scandinavian guidelines for initial management of adult patients with minimal, mild, and moderate head trauma, S100b was added as a clinical too $1^{14}$ : the biomarker, when sampled within 6 hours of the incident, can guide clinicians to decide between immediate computed tomography scan and/or observation under admission or discharge. ${ }^{14}$ Its prognostic value in neonatal and pediatric brain-injured populations is also under investigation. ${ }^{19}$ S100b has been studied as an early biomarker of perioperative brain injury. In 18 neonates undergoing early surgery for congenital heart disease on cardiopulmonary bypass, S100b increased during surgery and returned to baseline values postoperatively. ${ }^{5}$ Further, increased levels of S100b up to 48 hours after procedures have been associated with impaired neurodevelopment at 2 years of age. ${ }^{20}$ However, extracerebral contamination of $\mathrm{S} 100 \mathrm{~b}$ originating from mediastinal fat tissue or shed blood ${ }^{21}$ has been postulated to minimize the utility of $\mathrm{S} 100 \mathrm{~b}$ as a perioperative injury marker in this population. ${ }^{22}$

Neonatologists have investigated the prognostic value of $\mathrm{S} 100 \mathrm{~b}$ to predict neuromorbidity in neonates with hypoxic-ischemic encephalopathy (HIE). Compared with healthy controls, neonates with HIE expressed higher levels of S100b in umbilical cord blood within a few hours after birth. Furthermore, S100b correlates positively with the severity of HIE and the risk of permanent sequelae, ${ }^{23}$ and is increased in preterm infants who subsequently develop intraventricular hemorrhage. ${ }^{19}$ Asphyxiated infants with impaired neurodevelopment at 12-month follow-up have increased urine S100b levels during the first 72 hours of life. ${ }^{24}$ However, measurement of S100b has not yet been implemented as standard monitoring in at-risk neonates.

In the present study, mean baseline levels of $\mathrm{S} 100 \mathrm{~b}$ were higher in group $\mathrm{C}(0.94 \pm 0.38 \mathrm{mmol} / \mathrm{L})$ than in the intervention groups (HT, $0.67 \pm 0.25 \mathrm{mmol} / \mathrm{L} ; \mathrm{HC}$, $0.55 \pm 0.2 \mathrm{mmol} / \mathrm{L} ;$ HTC, $0.62 \pm 0.29 \mathrm{mmol} / \mathrm{L}$ ). This difference might be spurious and related to the low number of piglets $(n=9)$ in group C. Two of them showed comparatively high baseline levels of S100b (1.45 and 1.52 $\mathrm{mmol} / \mathrm{L}$ ), and mean levels excluding these 2 piglets $(0.78 \pm 0.22 \mathrm{mmol} / \mathrm{L})$ approximated to the $\mathrm{S} 100 \mathrm{~b}$ levels of the other groups. There was a decrease in serum albumin at Tr60 and post-Tr60 in the hypotensive groups, and this was likely because of hemodilution following replacement of withdrawn blood with Voluven to re-establish normovolemia.

The following should be considered when interpreting the results of the current study. Analysis of pig serum with a human S100b assay might have influenced the results. However, human S100b assays have previously been used in pigs, ${ }^{25}$ and are considered feasible because S100b is highly conserved among various animal species. ${ }^{26}$ Our study was performed over a period of 4 months and the serum analyses were performed following the inclusion of all animals. However, S100b concentrations have been found to be unaffected by storage time, so this is unlikely to have influenced our results. ${ }^{27}$ Further, S100b is not sensitive to hemolysis. ${ }^{11}$ In addition, samples were centrifuged onsite after being collected and frozen immediately. This minimized the risk of any further decay. ${ }^{27}$ Interventions and recovery were maintained for 60 minutes and the mean total duration of anesthesia ranged between 333 minutes (group HC) and 360 minutes (group HT). The elimination half-time of S100b ranges from $25 \mathrm{mi}-$ nutes ${ }^{28}$ to 200 minutes. ${ }^{29}$ As S100b is measurable in serum, 20 minutes following an insult, ${ }^{29}$ the timeframe used in the current study should be adequate to detect any change. Only piglets previously unexposed to GA were included. Male piglets are exposed to GA for castration at the age of 3 weeks, so only female piglets were used in the present study. This may be important and could have influenced our results; female piglets may respond differently to brain injury induced by hemorrhagic shock, with a reduced release of S100b compared with male animals. ${ }^{30} \mathrm{~A}$ piglet model was chosen because there is a good resemblance between the morpologic and neurophysiological development of the swine and human brain. ${ }^{6}$ However, piglets require higher doses of anesthetics to reach a sufficient depth of anesthesia compared with human infants. Hence midazolam infusion was added to ensure that the piglets had low muscle tone, did not shiver or breathe spontaneously. This does not reflect clinical practice and may limit the translational value of our results. 


\section{CONCLUSION}

In piglets exposed to stable GA maintained with sevoflurane and midazolam, serum levels of S100b steadily decreased over time. Hypotension, hypocapnia, or a combination of the 2 did not affect serum S100b levels; whether any S100b was released into cerebrospinal fluid is unknown. Consequently, this study does not support the hypothesis that S100b can be used as a biomarker of cerebral cell dysfunction in piglets under GA.

\section{REFERENCES}

1. Hansen TG. Use of anaesthetics in young children: consensus statement of the European Society of Anaesthesiology, the European Society of Paediatric Anaesthesiology, the European Association of Cardiothoracic Anaesthesiology and the European Safe Tots Anaesthesia Research Initiative. Eur J Anaesthesiol. 2017;34:327-328.

2. Michelet D, Arslan O, Hilly J, et al. Intraoperative changes in blood pressure associated with cerebral desaturation in infants. Paediatr Anaesth. 2015;25:681-688.

3. Ringer SK, Clausen NG, Spielmann N, et al. Effects of moderate and severe arterial hypotension on intracerebral perfusion and brain tissue oxygenation in piglets. Br J Anaesth. 2018;121:1308-1315.

4. Zongo D, Ribereau-Gayon R, Masson F, et al. S100-B protein as a screening tool for the early assessment of minor head injury. Ann Emerg Med. 2012;59:209-218.

5. Trakas E, Domnina Y, Panigrahy A, et al. Serum neurona biomarkers in neonates with congenital heart disease undergoing cardiac surgery. Pediatr Neurol. 2017;72:56-61.

6. Whitaker EE, Bissonnette B, Miller AD, et al. A novel, clinically relevant use of a piglet model to study the effects of anesthetics on the developing brain. Clin Transl Med. 2016;5:2

7. Kilkenny C, Browne WJ, Cuthill IC, et al. Improving bioscience research reporting: the ARRIVE guidelines for reporting animal research. PLoS Biol. 2010;8:e1000412.

8. Ringer SK, Ohlerth S, Carrera I, et al. Effects of hypotension and/or hypocapnia during sevoflurane anesthesia on perfusion and metabolites in the developing brain of piglets - a blinded randomized study. Paediatr Anaesth. 2016;26:909-918.

9. Hemphill JC III, Knudson MM, Derugin N, et al. Carbon dioxide reactivity and pressure autoregulation of brain tissue oxygen. Neurosurgery. 2001;48:377-383.

10. Clausen T, Scharf A, Menzel M, et al. Influence of moderate and profound hyperventilation on cerebral blood flow, oxygenation and metabolism. Brain Res. 2004;1019:113-123.

11. Thelin EP, Nelson DW, Bellander BM. A review of the clinical utility of serum S100B protein levels in the assessment of traumatic brain injury. Acta Neurochir (Wien). 2017;159:209-225.

12. Van Eldik LJ, Wainwright MS. The Janus face of glial-derived S100B: beneficial and detrimental functions in the brain. Restor Neurol Neurosci. 2003;21:97-108.
13. Kapural M, Krizanac-Bengez L, Barnett G, et al. Serum S-100beta as a possible marker of blood-brain barrier disruption. Brain Res. 2002;940:102-104.

14. Unden J, Ingebrigtsen T, Romner B. Scandinavian guidelines for initial management of minimal, mild and moderate head injuries in adults: an evidence and consensus-based update. BMC Med. 2013;11:50.

15. Jessen NA, Munk AS, Lundgaard I, et al. The glymphatic system: a beginner's guide. Neurochem Res. 2015;40:2583-2599.

16. Plog BA, Dashnaw ML, Hitomi E, et al. Biomarkers of traumatic injury are transported from brain to blood via the glymphatic system. $J$ Neurosci. 2015;35:518-526.

17. Jiang M, Sun L, Feng DX, et al. Neuroprotection provided by isoflurane pre-conditioning and post-conditioning. Med Gas Res. 2017;7:48-55

18. Wei H, Liang G, Yang H. Isoflurane preconditioning inhibited isoflurane-induced neurotoxicity. Neurosci Lett. 2007;425:59-62.

19. Gazzolo D, Di Iorio R, Marinoni E, et al. S100B protein is increased in asphyxiated term infants developing intraventricular hemorrhage. Crit Care Med. 2002;30:1356-1360.

20. Gunn JK, Beca J, Hunt RW, et al. Perioperative risk factors for impaired neurodevelopment after cardiac surgery in early infancy. Arch Dis Child. 2016;101:1010-1016.

21. Jonsson H, Johnsson P, Alling C, et al. S100beta after coronary artery surgery: release pattern, source of contamination, and relation to neuropsychological outcome. Ann Thorac Surg. 1999;68:2202-2208.

22. Johnsson P. S100-B in blood: a marker of brain damage or simply a covariate? Scand Cardiovasc J. 2000;34:548-549.

23. Zaigham M, Lundberg F, Olofsson P. Protein S100B in umbilical cord blood as a potential biomarker of hypoxic-ischemic encephalopathy in asphyxiated newborns. Early Hum Dev. 2017;112:48-53.

24. Gazzolo D, Marinoni E, Di Iorio R, et al. Measurement of urinary S100B protein concentrations for the early identification of brain damage in asphyxiated full-term infants. Arch Pediatr Adolesc Med. 2003; 157:1163-1168.

25. Ytrebo LM, Ingebrigtsen T, Nedredal GI, et al. Protein S-100beta: a biochemical marker for increased intracranial pressure in pigs with acute hepatic failure. Scand J Gastroenterol. 2000;35:546-551.

26. Larsson A, Lipcsey M, Sjolin J, et al. Slight increase of serum S-100B during porcine endotoxemic shock may indicate blood-brain barrier damage. Anesth Analg. 2005;101:1465-1469.

27. Raabe A, Kopetsch O, Gross U, et al. Measurements of serum S-100B protein: effects of storage time and temperature on preanalytical stability. Clin Chem Lab Med. 2003;41:700-703.

28. Jönsson H, Johnsson P, Höglund P, et al. Elimination of S100B and renal function after cardiac surgery. $J$ Cardiothorac Vasc Anesth. 2000;14:698-701.

29. Blomquist S, Johnsson P, Luhrs C, et al. The appearance of S-100 protein in serum during and immediately after cardiopulmonary bypass surgery: a possible marker for cerebral injury. J Cardiothorac Vasc Anesth. 1997;11:699-703.

30. Semenas E, Nozari A, Sharma HS, et al. Sex differences in cerebral injury after severe haemorrhage and ventricular fibrillation in pigs. Acta Anaesthesiol Scand. 2010;54:343-353.

Copyright (C) 2019 Wolters Kluwer Health, Inc. Unauthorized reproduction of this article is prohibited. This paper can be cited using the date of access and the unique DOI number which can be found in the footnotes. 